\title{
Interplay between p53 and non-coding RNAs in the regulation of EMT in breast cancer
}

\author{
Sergey Parfenyev ${ }^{1}$, Aastha Singh², Olga Fedorova ${ }^{1,3}$, Alexandra Daks $^{1,3}$, Ritu Kulshreshtha ${ }^{2}$ and Nickolai A. Barlev ${ }^{1,4,5}$
}

\begin{abstract}
The epithelial-mesenchymal transition (EMT) plays a pivotal role in the differentiation of vertebrates and is critically important in tumorigenesis. Using this evolutionarily conserved mechanism, cancer cells become drug-resistant and acquire the ability to escape the cytotoxic effect of anti-cancer drugs. In addition, these cells gain invasive features and increased mobility thereby promoting metastases. In this respect, the process of EMT is critical for dissemination of solid tumors including breast cancer. It has been shown that miRNAs are instrumental for the regulation of EMT, where they play both positive and negative roles often as a part of a feed-back loop. Recent studies have highlighted a novel association of p53 and EMT where the mutation status of p53 is critically important for the outcome of this process. Interestingly, p53 has been shown to mediate its effects via the miRNA-dependent mechanism that targets masterregulators of EMT, such as Zeb1/2, Snail, Slug, and Twist1. This regulation often involves interactions of miRNAs with IncRNAs. In this review, we present a detailed overview of miRNA/IncRNA-dependent mechanisms that control interplay between p53 and master-regulators of EMT and their importance for breast cancer.
\end{abstract}

\section{Facts}

- Epithelial-to-mesenchymal transition (EMT) is a highly orchestrated transcriptional program that takes place during the development and is mediated by several EMT-specific transcription factors (TFs): Zeb1/Zeb2, Snail, Slug, and Twist1.

- EMT is frequently re-activated during tumorigenesis, including breast cancer, and is responsible for drug resistance and metastasis.

- p53 hinders EMT by augmenting the expression of miRNA-200 that in turn, attenuates Zeb1/Zeb2 levels. The opposite is also true, thus forming a regulatory feedback loop.

\footnotetext{
Correspondence: Ritu Kulshreshtha (drritukulshreshtha@gmail.com) or Nickolai A. Barlev (nick.a.barlev@gmail.com)

${ }^{1}$ Institute of Cytology RAS, Saint-Petersburg 194064, Russia

${ }^{2}$ Department of Biochemical Engineering and Biotechnology, Indian Institute of Technology, Delhi 110016, India

Full list of author information is available at the end of the article

Edited by E. Candi
}

\section{Open questions}

- How does mutant p53 affect EMT in breast cancer?

- What are the relationships between p53, long noncoding RNAs, and miRNAs?

- Do p53-dependent miRNAs that target EMT:-TFs identified in various tumors also operate in breast cancer?

\section{Introduction \\ Breast cancer}

Breast cancer is the world's leading cause of cancerrelated death in women, impacting $>2.0$ million women each year worldwide. It is characterized by a high degree of heterogeneity on the clinical, morphological, and molecular levels. Despite its heterogeneity, breast cancer can be broadly categorized into four subtypes: luminal A (ER+, and/or PR+, HER2-), luminal B (ER+, PR+, HER2+), HER2 enriched (ER-, PR-, HER2+), and triple-negative breast cancers (TNBC) comprising basal-like (ER-, PR-, HER2-) and claudin-low breast cancers. The TNBC group is considered to be the most aggressive and highly 
metastatic type of breast cancer. Despite high incidences, the 5-year survival rate of women with breast cancer is comparatively favorable estimated at $\sim 80-90 \%$, but it rapidly decreases to around $24 \%$ in cases where distant metastases are detected. It is also predicted that $30 \%$ of early-stage breast cancer patients will progress to the severe forms of the disease, developing remote organ metastases ${ }^{1}$.

The aggressive, basal forms of breast cancer that are highly metastatic have undifferentiated, stem cell-like features correlating to a worse prognosis compared to other subtypes. This process of dedifferentiation and acquisition of an invasive phenotype is mediated by a molecular mechanism called epithelial-to-mesenchymal transition (EMT). The EMT process has been strongly correlated with tumor migration, invasion, and metastases. Since metastases are responsible for almost $90 \%$ of deaths in breast cancer, it is not surprising that a lot of effort has been made to study the molecular mechanisms governing the control of EMT and invasion.

\section{Non-coding RNAs in cancer}

Non-coding RNAs (ncRNAs) are RNA products transcribed from the non-protein-coding parts of the genome. Although originally believed to be "junk DNA," the evergrowing list of publications has defined the central role of expressed ncRNAs in development, differentiation, stress, and pathogenesis. There exists a vast variety of ncRNAs, including miRNAs, lncRNAs, piRNAs, and circRNAs, which efficiently modulate gene expression and importantly, regulate expression levels of each other. In cancers, these ncRNAs are reported to function either as oncogenes or tumor-suppressors depending on the cell-type and cellular context.

miRNAs are 18-24-nt long ncRNAs that primarily work by binding to short complementary sequences in $3^{\prime}$ UTRs of their target transcripts. This complementary binding usually results in the degradation or sequestration, and translation repression of the respective base-paired mRNAs. However, some evidence suggests that miRNAs can also be positive regulators of translation. By forming complexes with special proteins, such miRNAs on the contrary, have the potential to stabilize or upregulate the translation of their target transcripts in response to specific conditions and cues. Moreover, because of a short seed sequence, a single miRNA has the capacity to target mRNAs of multiple genes either from the same pathway or across diverse pathways, leading to global changes in the expression patterns, which are typically observed in malignancies. To date, there is a grate list of miRNAs, both intracellular and circulating, that were repeatedly demonstrated to be the biomarkers of $\mathrm{BC}$ progression, metastasis formation, drug resistance, and outcome prediction, e.g. miR-21, miR-155, or miR-1452.
LncRNAs are longer transcripts (>200 nt), and have diverse mechanisms of action. While the majority of lncRNAs are retained in the nucleus to perform tasks like epigenetic modifications, cis or trans regulation of gene expression, and splicing control, a number of lncRNAs are also translocated to the cytoplasm. These cytoplasmic lncRNAs act as competing endogenous RNAs (ceRNAs) and mediate the sponging of miRNAs via miRNA response elements (MRE), thereby eliminating the inhibition on miRNA target genes. They can also regulate translation by complexing with other proteins. The ability of IncRNAs to form secondary and tertiary structures allows them to exert various roles as scaffolds or decoys for their respective target proteins. In addition, lncRNAs can target other RNAs and metabolites, consequently interfering with gene expression. Like miRNAs, there has been a multitude of studies linking the dysregulation of lncRNAs to cancer. Thus, it was repeatedly shown that abnormal expression of numerous lncRNAs among which, for example, MALAT1, HOTAIR, and DANCR, contributes to $\mathrm{BC}$ aggressiveness, metastasis formation, and hence BC patients' prognosis outcome ${ }^{3}$. Therefore, owing to their clinical implications and networks of crosstalk, both miRNAs and lncRNAs are explored as therapeutic and diagnostic options in cancer and subjected to extensive research.

\section{Epithelial-to-mesenchymal transition (EMT)}

The epithelial-to-mesenchymal transition (EMT) is an evolutionary conserved reversible biological process that typically takes place during the development of organisms and results in a switch from epithelial phenotype to the mesenchymal phenotype. Morphologically, EMT is manifested in loss of cell polarity, adhesive, and tight contacts leading to the detachment of cells from the basement membrane. This is paralleled by massive cytoskeletal rearrangements that consequently change the morphology of epithelial cells to fibroblast-like, spindle-shaped cells and render them motile with an increased potential to invade the surrounding tissue and migrate to distant sites motile with an increased potential to invade the surrounding tissue and migrate to distant sites ${ }^{4}$. While EMT is utmost essential during embryo-development (classified as EMT type-1) and wound healing (EMT type2 ), it plays an equally important role in cancer progression and dissemination (EMT type-3), consequently determining the prognosis. Mesenchymal cells produced through EMT have been found to be similar to tumorinitiating CSCs with a high expression of CD44, exhibiting the drug-, apoptosis-, and anoikis-resistant phenotypes ${ }^{5,6}$. Further, these cells are able to avoid oncogene-induced senescence and are immunosuppressive in nature ${ }^{7}$.

It is also worth noting that EMT has a large degree of plasticity, i.e. it can be reversed. This process is called 
mesenchymal-to-epithelial transition (MET). These evidences support the notion that EMT not only helps cancer cells to invade and metastasize but also confers an enhanced endurance and survival, enabling them to establish new tumors at distant sites.

EMT is activated and stabilized in response to a number of paracrine signals driven by stromal cells, such as fibroblasts, myofibroblasts, and mesenchymal stem cells. These signals and growth factors trigger cascades inside the target cells that culminate in the activation of gene expression pathways that initiate EMT. Cancer cells exploit this program to their benefit by modulating a handful of core transcription factors involved in the direct regulation of EMT, conveniently known as the EMT-TFs. These core EMT-TFs, which include Zeb1, Zeb2, Snail, Slug, and Twist1, are pleiotropic in nature and act in various combinations in different cell types to kickstart EMT. In recent times, non-coding RNAs (miRNAs and lncRNAs) have been discovered to act as critical downstream mediators by modulating a large number of target genes and pathways together to either promote or suppress $\mathrm{EMT}^{8}$.

It has also been discovered that breast cancer cells of the basal phenotype are more primed transcriptionally to respond quickly to extracellular EMT-inducing signals like TGF- $\beta$, thereby becoming more mesenchymal and dedifferentiated as compared to luminal cells which resist this transition ${ }^{9}$. This indicates that the phenotype of the cell is also an important determinant of its response to EMTinducers and may explain why basal-like breast cancers are so aggressively metastatic with a bleak prognosis.

\section{The master-regulators of EMT in breast cancer}

As mentioned above, the EMT program is largely regulated at the level of gene expression by several transcription factors, including Zeb1 (Zfhx1a, Bzp, Zfhep, dEF1, TCF8, Nil-2, and AREB6) ${ }^{10}$, Zeb2 (Zfh1, CIP1) ${ }^{11}$, Snail (SNAI1), and Slug (SNAI2) ${ }^{12}$, as well as Twist $1^{13}$. Twist1 belongs to the family of Helix-Loop-Helix (HLH) factors that bind specific DNA sequences called E-box (5'-CANNTG-3') that are located in the regulatory regions of their target genes. In response to specific signaling cues, they repress transcription of the epithelial genes and promote the expression of mesenchymal genes.

The expression of Zeb1 is undetected in luminal breast cell lines but is observed in basal ones with the highest expression in CD44 ${ }^{\text {high }}$ cancer stem cells $(\mathrm{CSC})^{9}$. Similarly, while Snail is detected in all subtypes, its expression is also the highest in basal-like cells ${ }^{14}$. A study shows that di-acetylated Twist1 is responsible for activating WNT5A and directly contributes to the invasion and tumorigenicity of basal-like breast cells ${ }^{15}$. Likewise, Slug is also known to suppress the expression of ER-alpha by binding to the E-box motif and is responsible for the migration of triple-negative MDA-MB-231 cells ${ }^{16}$. Cells overexpressing Slug also display the basal-like phenotype ${ }^{17}$. These studies reinforce the concept that the EMT pathway is majorly responsible for driving the malignancy of basal-like and TNBC cells, leading to dismal outcomes.

\section{Association of p53 and EMT}

The p53 tumor suppressor, known as the "genome guardian", is known to play a key role in preventing tumor development ${ }^{18}$. The p53-coding gene TP53 is mutated in the vast majority of human tumors (in more than $50 \%$ of cases). p53 plays the role of a transcription factor that acts in response to various stress signals, causing cell cycle arrest, cell aging, and apoptosis, as well as controlling the metabolism and antioxidant status of cells ${ }^{19}$. Functioning in the development, p53 limits the plasticity of epithelial cells during EMT. For example, p53 can interfere with the delamination of the neural crest, which usually occurs as a result of triggering $\mathrm{EMT}^{20}$.

The published studies of the past decade highlighted the novel role of p53 in regulation of metastasis. Wildtype p53 (WTp53) was shown to prevent EMT and the associated stem cell-like phenotype across multiple cancers. As a transcriptional factor, p53 can repress EMT by helping the cells maintain the epithelial gene signature. Furthermore, p53 was shown to induce the attenuation of EMT-TFs levels ${ }^{21}$ via the augmentation of the expression of EMT-suppressing miRNAs. In this respect, it is important to note that p53 induces the expression of miRNAs that target the Zeb, Snail, and Twist families of transcription factors ${ }^{4}$ (Figs. 1, 2). In turn, EMT regulators are shown to attenuate the p53 functions. For example, one of the key EMT inducers Snail) is known to bind to and repress wild-type p53 directly. This Snail-mediated inhibition of p53 was found to be essential for tumor-initiation and growth in breast cancer models ${ }^{22}$.

The importance of p53 in averting cancer metastasis is evident in cases where p53 is lost or worse, mutated. Loss of WTp53 in breast epithelial cells triggers EMT with a parallel increase in the number of cancer stem cells and is associated with a higher tumor grade. This has been attributed to the decrease in expression of tumor-suppressing microRNAs such as miR-200c, which are directly transactivated by $\mathrm{p} 53^{23}$.

On the other hand, the presence of gain-of-function (GOF) mutant p53 is enough to unleash EMT and makes the cells more migratory in nature. Mutant p53 upregulates the expression of EMT-TFs like Twist1 and Zeb1 ${ }^{24}$. This likely occurs because many forms of mutant p53 retain their transcriptional activity albeit with different sequence-specificity ${ }^{25}$. Furthermore, GOF mutants of p53 can mediate its oncogenic effects via the regulation of onco-miRs. This also explains why only the mutant p53 is 


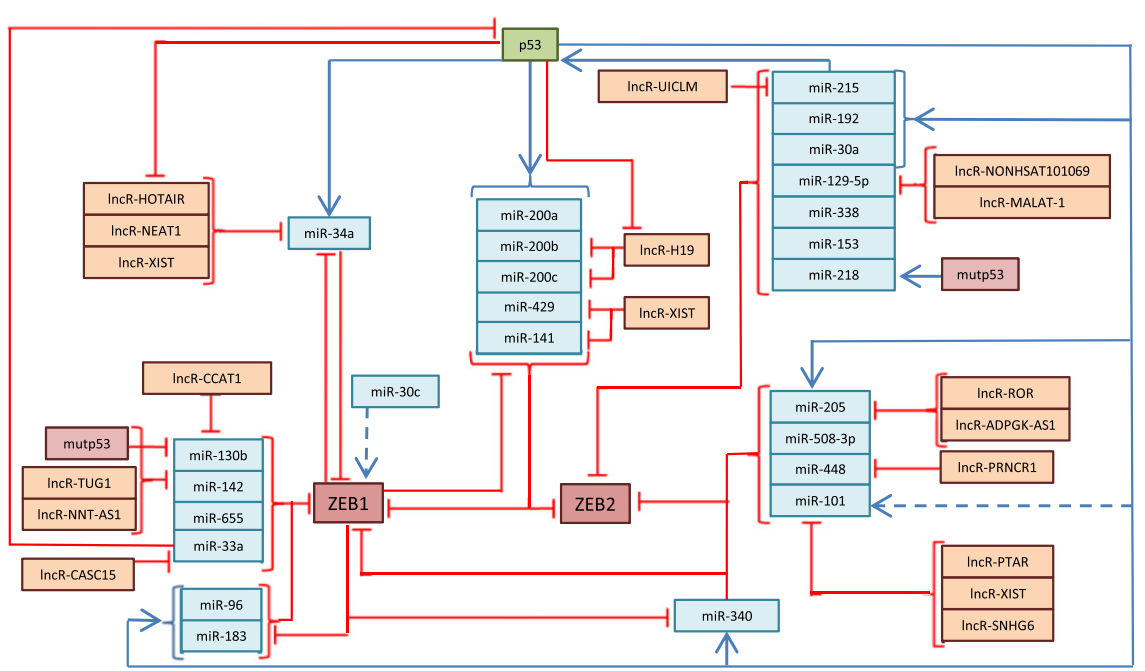

Fig. 1 Cross-regulation of p53 and Zeb1/2 EMT-TFs in breast cancer (BC) via miRNAs and IncRNAs. p53 mediates expression of the indicated miRs (denoted with blue color) that in turn target Zeb1/2 EMT-TFs (denoted as burgundy-colored boxes). Long non-coding RNAs (IncRNAs) denoted with pink-color act as competing endogenous RNAs (ceRNAs) to counteract the effect of miRs. Interactions between all of these players form simple links and feedback loops (indicated with arrows and bars). Positive influence is denoted by blue arrows and negative is denoted by red bars.

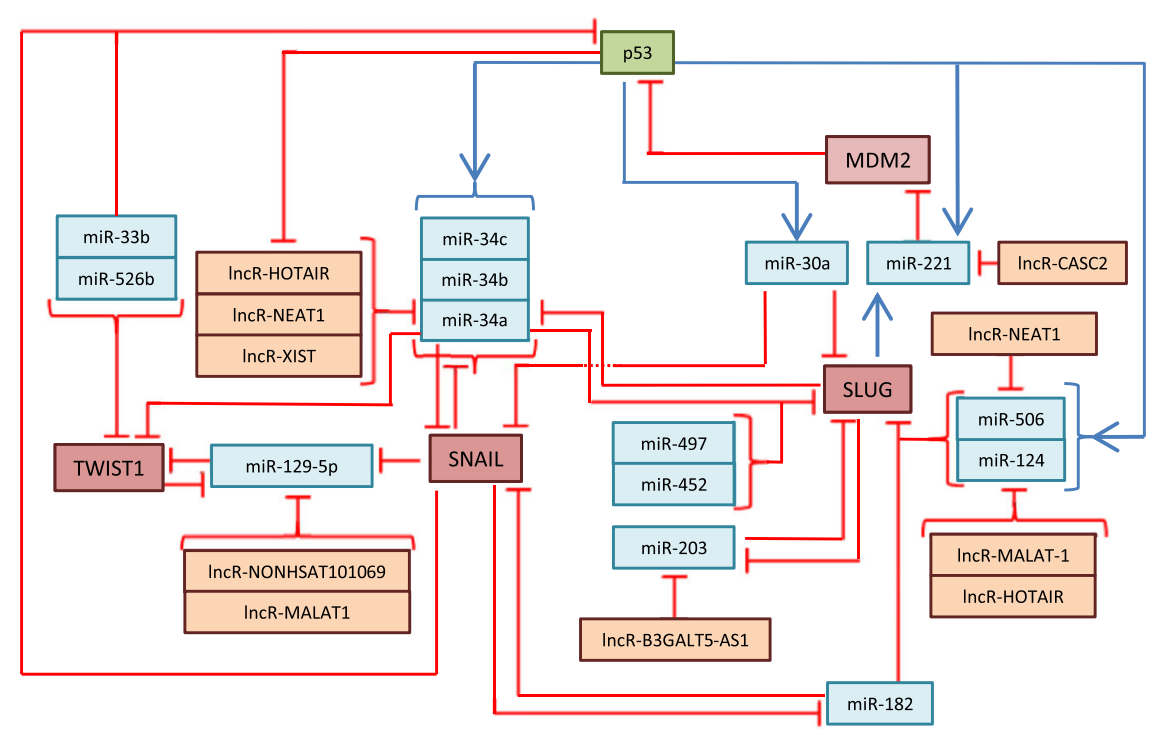

Fig. 2 Interactions between p53 and MDM2 in respect to EMT-TFs Twist, Snail, and Slug, which are mediated by miRNAs and IncRNAs in breast cancer. The color-coding is the same as in Fig. 1.

able to activate multiple oncogenic miRNAs like miR-155 in breast cancer.

Control over the epithelial cell phenotype between p53 and EMT-TFs is a dynamic two-way process. To counteract the repressive effect of p53 on EMT, the EMT-TFs themselves negatively regulate p53 and its regulated miRNAs. For example, Twist1 is known to attenuate the effects of p53 and inhibit cellular apoptosis. Likewise, Snail directly binds and represses $\mathrm{p} 53^{22}$. As a result of such opposing effects, multiple feedback loops have evolved that include intricate networks of p53, EMT-TFs, and non-coding RNAs. In this review, we attempt to portrait this complex circuitry.

\section{p53-regulated miRNAs in control of EMT TFs}

p53 has been shown to induce the expression of a number of miRNAs that suppress EMT via targeting various EMT-regulating transcription factors, such as Zeb1, Zeb2 ${ }^{4}$, and Snail ${ }^{26}$. A list of various miRNAs is shown in Tables 1-3. 


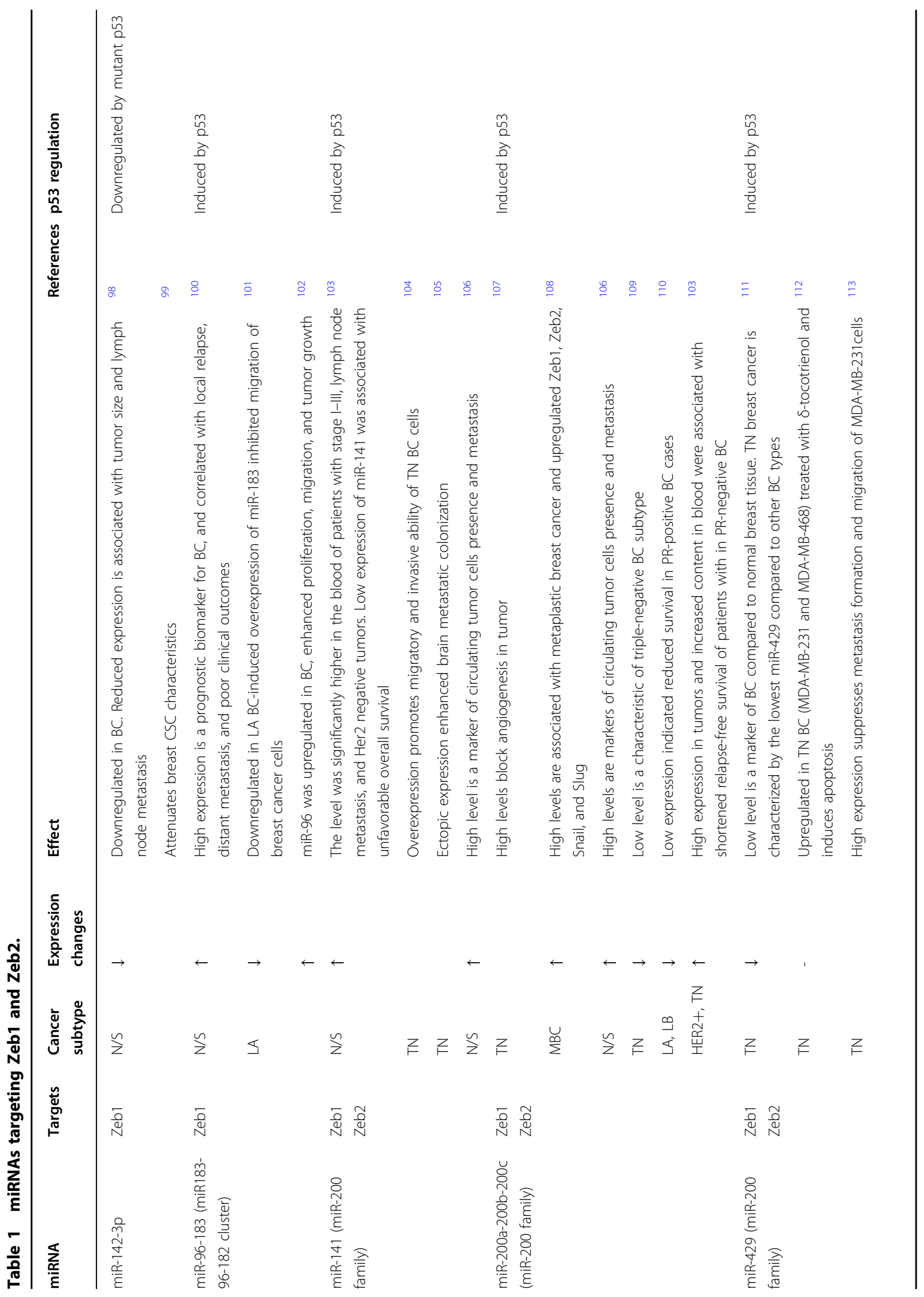




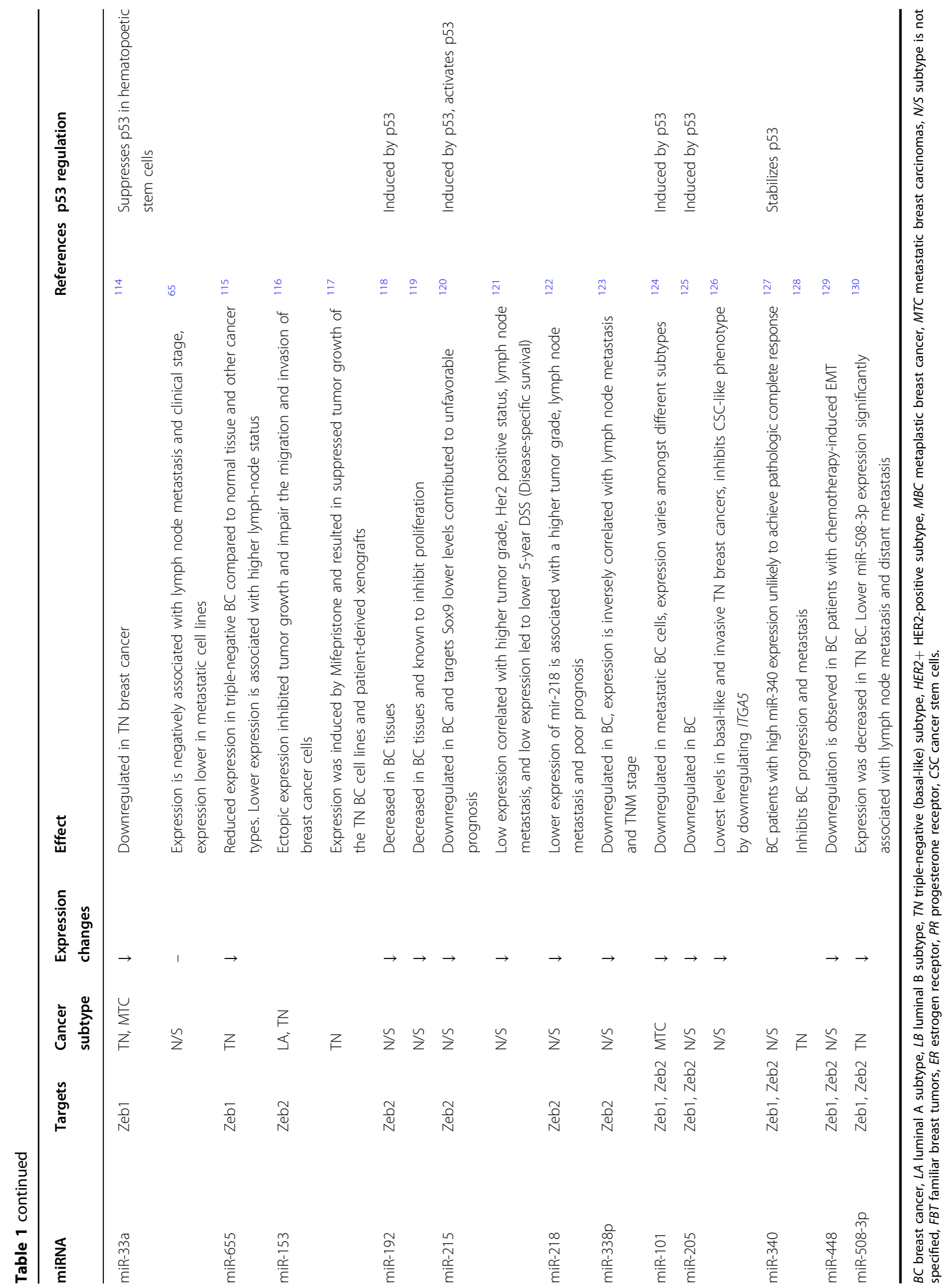




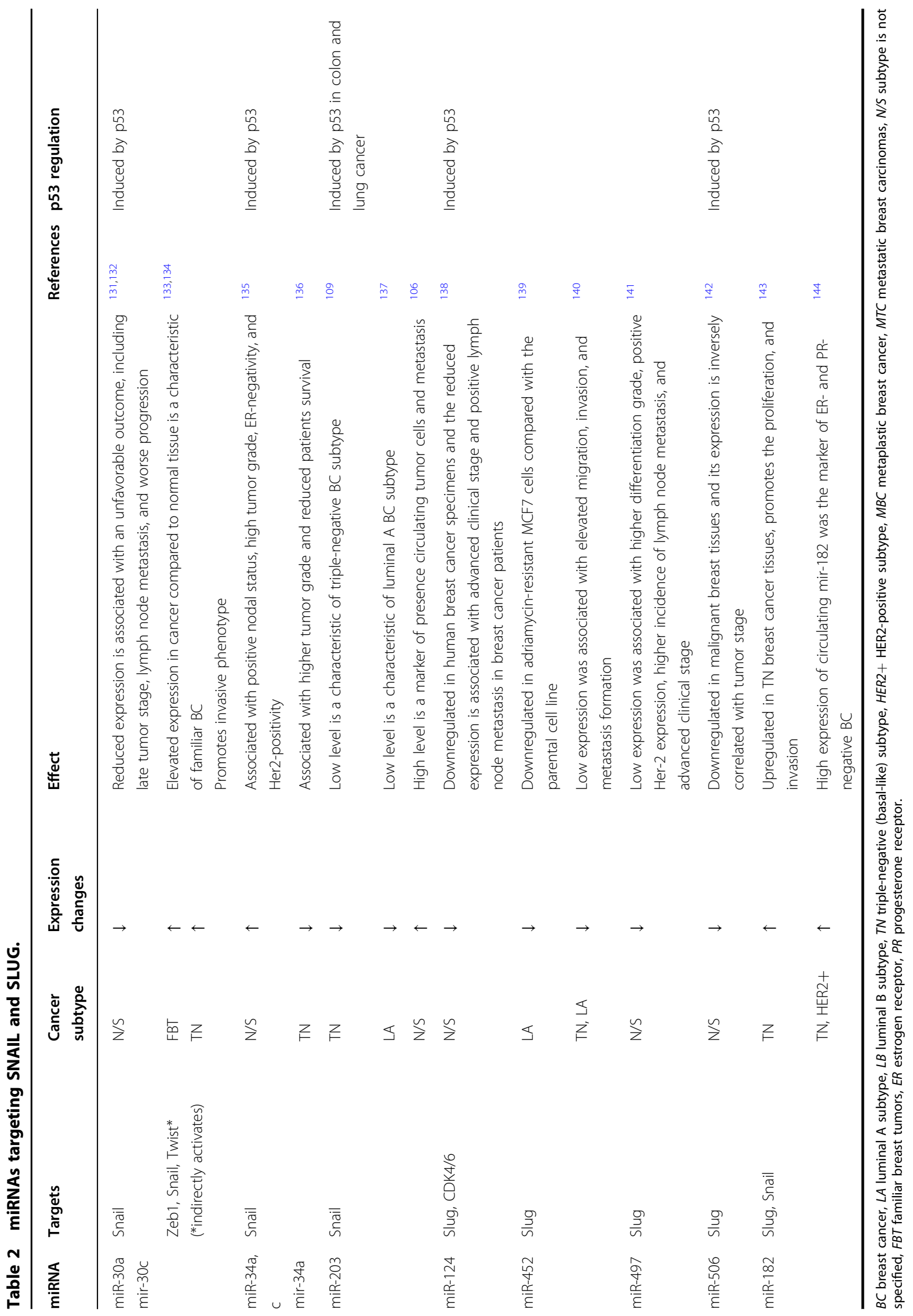




\section{miR 200 family}

Kim et al. were the first to show that p53 brings about the suppression of EMT by inhibiting the expression of Zeb1 and Zeb2 through induction of the miR-200 family of miRNAs in hepatocellular carcinoma ${ }^{4}$. Later, Chang and colleagues found that the p53 family directly controls the expression of the miR-200 family in breast cancer ${ }^{23}$.

Several studies have now established that miR-200 family acts a critical mediator of the p53-regulated suppression of EMT by targeting several EMT-TFs including Zeb1 to maintain the epithelial phenotype. All five members of the miR-200 family (miR-200a, miR-200b, miR-200c, miR-141, and miR-429), as well as miR-205, were shown to suppress EMT via targeting Zeb1 ${ }^{27}$. The increase in ectopic expression of the genes of this family (miR-200a, miR-200b) leads to morphological changes in cells, which change from a spindle-shaped mesenchymal back to a round epithelial form with concomitant association of cells into groups. In turn, ablation of miR-200 promoted chemoresistance of breast cancer cells during $\mathrm{EMT}^{28}$. Loss of the miR-200 family in regions of metaplastic breast cancer specimens was shown to be paralleled with loss of E-cadherin. Thus, downregulation of the members of this miR family is likely to be an important step in the tumor progression.

\section{Regulation by EMT-TFs: Zeb1 and Snail}

Zeb1, along with Snail, plays a critical role in the regulation of p53-regulated miR-200 family members. Zeb1 and Snail have been reported to bind the promoters of miR-200b-200a-429, miR-200c-149, and curb their expression $^{29}$. Additionally, Snail is also involved in promoting the methylation of miR-200 locus, which is essential for sustaining the mesenchymal phenotype in cancer cells.

\section{Regulation of miR-200 family by IncRNAs}

Several p53-suppressed long non-coding RNAs are also involved in the regulation of the miR-200 family, including lncRNA H19 and lncRNA XIST ${ }^{30,31}$. Both lncRNA H19 and lncRNA XIST act as so-called competing endogenous RNAs (ceRNAs), which physically associate with specific miRs through miRNA response elements (MREs) on their target transcripts ${ }^{32}$. Thus, lncRNA H19 and lncRNA XIST inhibit miR-200b and miR-200c $\mathrm{c}^{33}$, miR- $141^{30}$ and miR- $429^{31}$, respectively.

Overall, the expression of the miR-200 family seems to be under tight regulation by TFs, epigenetic regulators and lncRNAs, and displays a strong effect on the EMT process by targeting the EMT TFs.

\section{miR-34}

It has recently been shown that miR-34a, another target of $\mathrm{p} 53^{34}$ can directly suppress Zeb1. p53 augments the 
expression of miR-34a both in cultured cells, as well as in irradiated mice ${ }^{35}$. Notably, Zeb1 can reciprocally inhibit the transcription of miR-34 by binding to the E-boxes located in the promoter regions of miR-34a-b-c ${ }^{36}$. In addition to targeting Zeb1, miR-34a also downregulates Snail, Slug, Twist1, and Notch in metastatic breast cancers $^{37}$, as well as other stemness-associated factors like CD133, CD44, BMI1, and $\mathrm{c}-\mathrm{Myc}^{36}$ via direct binding $3^{\prime}$ UTRs of the respective genes and thereby attenuating the process of EMT and metastasis. Given that miR-34 targets several critical EMT-TFs, it can be considered as a "universal weapon" against EMT.

\section{Regulation of miR-34 by EMT-TFs: Zeb1, Snail, and Slug}

Similar to the miR-200 regulatory mechanism described above, Zeb1 and Snail regulate miR-34a. Siemens and colleagues have established the presence of a negative feedback loop, wherein miR-34a and Snail repress each other by direct binding. This interplay is mediated via the p53-dependent activation of miR-34a during the regulation of $\mathrm{EMT}^{36}$. Another member of the Snail family, SLUG has also been reported by De Carolis et al. to directly bind to the promoter of miR-34a and repress its transcription in breast cancer cells exposed to hypoxia, and upregulate the expression of Carbonic Anhydrase isoenzyme 9 (CAI9). This enabled the cells to acquire a stemness like phenotype with an increased ability to form mammospheres $^{38}$.

\section{Regulation of miR-34 by IncRNAs}

It has been noted that several lncRNAs, including lncRNA XIST ${ }^{39}$ and the p53-inducible LncRNA NEAT $1^{40}$ act as ceRNA for miR-34a, which leads to the activation of EMT. The physiological meaning of LncRNA NEAT1 activation by p53, especially in breast cancer, needs to be investigated further.

\section{miR-192/215 family}

Kim et al. demonstrated that the p53-regulated miR-192 was able to prevent EMT in hepatocellular carcinoma by suppressing Zeb2 at the post-transcriptional level ${ }^{41}$. Similarly, in the same study, another p53-dependent miR215 was shown to significantly reduce levels of mRNA and protein of Zeb2 in cells of the colorectal cancer lines SW620 and DLD-1. Importantly, the observation of EMTsuppressing activity of miR-215 was confirmed in breast cancer cells ${ }^{42}$. However, in this case, the authors focused on Sox-9 as the main target of miR-215. The question of whether miR-215-dependent attenuation of Zeb2 contributed to this process remains to be answered.

\section{Regulation of miR-215 by IncRNAs}

Interestingly, LncRNA-UICLM was shown to compete with miR-215 thereby regulating the expression of Zeb2.
lncRNA-CDC6, which is overexpressed in breast cancer tissues, also negatively regulates miR-215 by sponging it. This inhibition of miR-215 promoted the proliferation and migration ability of breast cancer cells ${ }^{43}$.

\section{miR-30 family}

The miR-30 family, which includes five members (miR30a-e), is involved in the pathogenesis of various types of tumors. It has also been demonstrated that $\mathrm{p} 53$ binding to the miR-30a promoter induces the transcription of both miRNA chains $-5 \mathrm{p}$ and $3 \mathrm{p}-$ which are able to interact with Zeb2 $2^{44}$. Interestingly, the same family members can be either cancer-promoting or tumor-suppressing. For example, miR-30d $\mathrm{d}^{45}$ and miR-30a have been identified as anti-metastatic factors in different tumors. In an experiment conducted by Mahsa and colleagues using MCF-7 breast cancer cells, an inverse correlation was found between miR-30c and Zeb1. This suggests that miR-30c likely activates Zeb1, although this has not been confirmed by direct experiments ${ }^{46}$. Furthermore, reduced miR-30 expression has been reported to be critical for the maintenance of self-renewal and inhibition of apoptosis in breast tumor-initiating cells.

\section{miR-124}

Mutation in or deletion of the TP53 gene affects the expression of several miRNAs, among which miR-124 was the most strongly downregulated. This effect correlated with an upregulation of the anti-apoptotic gene, iASPP, suggesting that the latter was the target for miR-124. Consistent with this notion, p53 was shown to bind the promoter of the miR-124 gene to facilitate its expression, which consequently inhibited iASPP expression. Overexpression of miR-124 led to suppression of the CDK4 protein expression and attenuated cell viability, proliferation, and cell cycle progression in MCF-7 and MDAMB-435S breast cancer cells in vitro ${ }^{47}$.

\section{miR-124 targets SLUG}

The expression of miR-124 is reduced in human breast cancer tissues and its levels have been inversely correlated with the tumor grade. miR-124 attenuated the migration of metastatic breast cancer cell line MDA-MB-231 and reversed the morphology from spindle-shaped to epithelial cobblestone-like, with a parallel increase in the expression of E-cadherin. It was found that miR-124 mediated direct targeting of Slug's 3'UTR was responsible for the reversal of EMT characteristics ${ }^{48}$.

\section{Regulation of miR-124 by IncRNAs}

LncRNA MALAT-1 serves as a ceRNA for miR-124 in breast cancer ${ }^{47}$ and in the development of non-small cell lung cancer. MALAT1 eliminates the suppressive effect of miR-124 on CDK4 and increases cell proliferation. 
In addition, lncRNA HOTAIR can also act as ceRNA for miR-124. The authors suggest that HOTAIR activation may enhance the EMT process through inhibition of miR- $124^{49}$.

\section{miR-203}

An important tumor-suppressive miRNA, miR-203, is known to inhibit the invasiveness and migration of breast cancer cells. Two independent studies have shown that miR-203 also targets Slug directly through its $3^{\prime} \mathrm{UTR}^{50}$. Ding et al. described the presence of a double-negative feedback loop, wherein the promoter of miR-203 itself is targeted and repressed by Slug. In a similar manner, Slug also directly suppresses the expression of members of the miR-200 family ${ }^{51}$. Recently, miR-124 and miR-203 were shown to inhibit the expression of Zeb2 at the posttranscriptional level in human kidney carcinoma cells ${ }^{52}$. It will be interesting to see whether miR-203 can also attenuate the expression of Zeb2 and potentially Zeb1 in metastatic breast cancer cell models.

Studies on keratinocytes have demonstrated the expression of miR-203 to be dependent on p53. In these cells, the knockdown or HPV-mediated degradation of p53 significantly decreased the level of mir-203, while activation of p53 by doxorubicin resulted in the opposite effect $^{53}$. This observation of mir-203's dependence on p53 was also confirmed in lung cancer cells, wherein the reintroduction of p53 in p53-null cells increased miR-203, while mutant p53 failed to do so. Consequently, the overexpressed miR-203 augmented the sensitivity of both colon and lung cancer cells to gemcitabine-induced apoptosis, possibly by positively regulating the expression of Puma. ${ }^{54}$. Although the association between p53 and miR-203 has not been explored in breast cancer yet, it is very tempting to speculate that such regulation also takes place in $\mathrm{BC}$ cells.

\section{Regulation of miR-203 by IncRNA}

LncRNA B3GALT5-AS1, which promoted colon cancer invasion, inhibited miR-203 directly by interacting with the miR-203 promoter ${ }^{55}$. The overexpression of this lncRNA thereby increased the cellular levels of Zeb2 and Slug and promoted EMT.

\section{miR-129-5p}

Tan et al. have reported that the promoter region of miR-129-5p could be potentially targeted by p53, whose malfunction is frequently detected in human cancers ${ }^{56}$. High levels of miR-129-5p increased the expression of E-cadherin by interacting with the $3^{\prime} \mathrm{UTR}$ region of Zeb2 ${ }^{57}$. miR-129-5p levels were significantly decreased in breast cancer cell lines. In a study by Yu et al., Twist1 was shown to be a direct target of miR-129-5p and was repressed on its overexpression. The promoter of
miR-129-5p reportedly contains three E-box motifs, and the ChIP analysis confirmed the enrichment of both SNAIL and TWIST1 to these sites, correlating with a decrease in the promoter activity. This suggests the presence of reciprocal negative regulation between Twist1 and $\mathrm{miR}-129-5 \mathrm{p}$. This downregulation of miR-129-5p via the Twist1-Snail feedback loop stimulates EMT and is associated with poor prognosis in breast cancer ${ }^{58}$.

\section{Regulation of miR-129 by IncRNAs}

The lncRNA NONHSAT101069 was found to function as ceRNA by directly binding to miR-129-5p in breast cancer cells ${ }^{59}$. IncRNA MALAT1 also targets miR-129-5p by directly binding and sponging it, resulting in greater cell invasion and migration. MALAT1 and miR-129-5p were found to have an inverse correlation of expression in TNBC tissues ${ }^{60}$. There have been numerous other reports of MALAT1 regulating multiple modulators and enhancing the EMT and stemness phenotype, which underlines its importance in breast cancer progression ${ }^{61}$. Interestingly, it was found that mutant gain-of-function p53, along with other factors ID4 and SRSF1, associates with MALAT1, and represses the production of an antiangiogenic splicing isoform of VEGFA, hence promoting angiogenesis in breast cancer ${ }^{62}$.

\section{The miR-33 family miR-33a-5p targets Zeb1}

Surprisingly, miR-33a-5p, which was shown to target p53 in stem cells ${ }^{63}$, also inhibits the expression of Zeb1 by interacting with its $3^{\prime} U T R$ region $^{64}$. The overexpression of miR-33a in metastatic breast cancer cells remarkably decreases cell proliferation and invasion in vitro and significantly inhibits tumor growth and lung metastasis in vivo. Conversely, its knockdown in non-metastatic breast cancer cells considerably enhances cell proliferation and invasion in vitro and promotes tumor growth and lung metastasis in vivo, which strongly supports the idea of miR-33 as a tumor suppressor ${ }^{65}$. In hematopoietic stem cells, it is known that miR-33 negatively regulates p53 by binding to two conserved motifs in the $3^{\prime}$ UTR of its mRNA. This downregulation of p53 and p53-activated apoptosis is responsible for maintaining the stemness phenotype, and reveals the molecular mechanisms of how miR-33 plays opposing roles depending on the cell type ${ }^{63}$.

\section{miR-33b targets Twist1}

Twist 1 is overexpressed in aggressive breast cancers and is also known to promote breast cancer metastasis to the bone. Lin et al. reported that the levels of miR-33b in human breast cancer tissues are significantly reduced and correlate inversely with node metastasis and tumor stage ${ }^{66} . \mathrm{MiR}-33 \mathrm{~b}$ was shown to directly bind to the $3^{\prime} \mathrm{UTR}$ of Twist1 and suppress it. Furthermore, ectopic overexpression of miR-33b 
in metastatic breast cancer cell lines decreased the number of cancer stem cells (CSCs) and their invasive properties. Their ability to metastasize was also found to be reduced in mice models in vivo, suggesting that this miR plays a vital role in maintaining the stemness and invasion properties of breast cancer cells ${ }^{66}$.

\section{Regulation of miR33a-5p by IncRNA}

Using pull-down analysis with biotin-labeled miR-33a$5 p$ in gastric cancer cells, it was found that miR-33a-5p directly interacted with lncRNA CASC15, establishing its role as a ceRNA for miR-33a-5p ${ }^{64}$. RT-PCR and Western blot analyzes proved that the inhibition of IncRNA CASC15, as well as the induced expression of miR-33a$5 \mathrm{p}$, reduced levels of Zeb1 expression in AGS and SGC7901 gastric cancer cells ${ }^{64}$.

\section{Other miRNAs}

\section{miR-101}

miR-101 is one of the potential targets of p53 in induced pluripotent stem cells ${ }^{67}$. Several studies implicate miR101 as a regulator of breast cancer, which targets several important oncogenes. By binding to the $3^{\prime} \mathrm{UTR}$ of the Zeb1 and Zeb2 mRNA sites, it reduces the levels of Zeb1 and Zeb2, which leads to the attenuation of EMT ${ }^{68}$.

The IncRNA PTAR (a pro-transition associated RNA, which is upregulated in mesenchymal subtype cells) has been discovered to inhibit miR-101 activity, acting as ceRNA, and eventually promoting $\mathrm{EMT}^{48}$. In addition, lncRNA SNHG6 ${ }^{69}$ and lncRNA XIST ${ }^{70}$ also inactivate miR-101 by the same mechanism.

\section{Regulation of miR-101 by IncRNAs}

The lncRNA PTAR (a pro-transition associated RNA) has been discovered to inhibit miR-101 activity, acting as ceRNA and eventually promoting $\mathrm{EMT}^{71}$. In addition, another lncRNA SNHG6 ${ }^{69}$ and lncRNA $\mathrm{XIST}^{70}$ also inactivate miR-101 by the same mechanism.

\section{miR-205}

In breast cancer, p53-inducible miR-205 was shown to possess tumor-suppressive functions ${ }^{72}$. A team of scientists led by Lee ${ }^{73}$ showed that miR-205 inhibits both Zeb1 and Zeb2 mRNAs in the breast cancer cell lines MCF7, MDA231, and SK-BR-3. However, under hypoxic conditions miR-205 in lung cancer promoted EMT by targeting apoptosis-stimulating protein of p53-2 (ASPP2) ${ }^{74}$. This fact indicates that depending on the environment, miR205 can play either a positive or negative role in the regulation of EMT.

miR-205-5p is under the regulatory control of lncRNAs $\mathrm{ROR}^{75}$ and ADPGK-AS1 ${ }^{76}$ that inhibit miR-205-5p by acting as ceRNA, promoting cell proliferation, migration, and invasion through stabilizing mRNA of Zeb1.
LncRNA-ROR is upregulated in breast tumors, and forced expression of lncRNA-ROR in breast epithelial cells leads to visible changes in morphology, increases mesenchymal markers, activates EMT, and promotes invasion, further generating stem-cell-like cells $\left(\mathrm{CD} 44^{\mathrm{hi}} / \mathrm{CD} 24^{\mathrm{lo}}\right)$ with advanced mammosphere forming ability ${ }^{75}$.

\section{miR-221}

miR-221 is well-known as a basal subtype-specific miRNA and is overexpressed in TNBC cells. It targets the tumor suppressor p27KIP ${ }^{77}$. Noteworthy, in metastatic MDA-MB-231 cells, the miR-221 gene expression was upregulated by Slug, as it was recruited to the E-boxes located in the miR-221 promoter. Conversely, repression of Slug led to decreased levels of miR-221, as well as decreased cell motility ${ }^{78}$.

miR-221 was reported to activate the $\mathrm{p} 53 / \mathrm{mdm} 2$ axis by inhibiting Mdm2. In turn, p53 activation was shown to enhance miR-221 expression ${ }^{79}$.

It is important to note that this effect was evident only upon activation of $\mathrm{p} 53$ by the cytotoxic drug, doxorubicin. Future studies should elucidate whether the p53activating effect of miR-221 is still valid in metastatic EMT cells.

In hepatocellular carcinoma, miR-221 (as well as miR-24) is negatively regulated by the sponging action of CASC2, which is a well-established tumor-suppressive $\operatorname{lncRNA}{ }^{80}$. It was found that by inhibiting miR-221, CASC2 could mediate the sensitivity of cancer cells to apoptosis induced by anti-cancer agent TRAIL and upregulated Caspase-3, which is a direct target of miR-221.

\section{miR-506}

MiR-506 was shown to target the Slug gene directly and decrease EMT characteristics in breast cancer cell lines ${ }^{81}$. The upstream region of miR-506 has a putative p53 response element. p53 was shown to directly target and upregulate its expression in lung cancer cells $^{82}$, but no such study has been carried out yet for breast cancer.

MiR-506 is also known to induce the demethylation of the MEG3 promoter of a lncRNA MEG3 via SP1/SP2 and Dnmt1. MEG3 is a lncRNA that inhibits cell growth and metastasis of breast cancer cells ${ }^{83}$. The expression of lncRNA MEG3 is reduced in breast cancer tissues and also in cell lines MCF7 and MDA-MB-231. Overexpression of MEG3 also led to a decrease in the Mdm2 RNA and protein levels, which subsequently stabilized p53 on the protein levels resulting in activation of its targets including $\mathrm{p} 21$, Maspin, and $\mathrm{KAI}^{84}$. Another lncRNA that plays an important role in regulating miR506 is lncRNA NEAT1. The gene expression profile data and results of dual-luciferase reporter assay in serous ovarian cancer demonstrated that lncRNA NEAT1 
functioned as a competing ceRNA for miR-506 to promote cell proliferation and migration ${ }^{85}$.

\section{The H19/miR-675 locus}

Another long non-coding RNA, LncRNA H19, is highly expressed in metastatic breast cells. The promoter region of LncRNA H19 has been shown to be effectively suppressed by the wild-type p53 protein ${ }^{86}$. Slug upregulates the expression of the $\mathrm{H} 19$ locus. Interestingly, this locus also encodes for another miRNA, miR-675. It was found that H19 itself could also upregulate Slug through a mechanism dependent on miR-675. This positive feedback loop increased the invasive properties of cancer cells both in vitro and in vivo ${ }^{87}$.

\section{miR-10b}

MiR-10b is a well-known metastatic miRNA in breast cancer, which is highly expressed in breast cancer tissues and metastatic cell lines. While it was found to have no significant effect on cellular proliferation, the invasiveness of cells, both in vitro and in vivo, significantly increased on miR-10b ectopic expression, and distant metastasis was also promoted. E-box motifs were discovered in the region upstream of miR-10b, and Twist1 was shown to directly bind to one of these regions. Because of this direct binding and their positive correlation of expression, it was suggested that Twist 1 drives the expression of miR-10b to promote cancer spread. Downstream, miR-10b targets HOXD10, which is involved in the repression of cell motility genes ${ }^{88}$. However, miR-10b promoter has a p53 response element, and Bisio et al. showed using CHIP assay that p53 was actively recruited to the miR-10b promoter in MCF7 cells and induced its expression ${ }^{89}$. This seems counter-intuitive to the role of miR-10b as a "metastamiR", and functional studies are lacking to further explore the effects of this association.

Hence, the existence of multiple feedback loops between the EMT-TFs and p53-dependent miRNAs may be responsible for driving the final fate of the cell.

\section{miRNAs regulated by mutant p53 in EMT}

Since wild-type and mutant p53 control different networks of genes, it is not surprising that they also affect different spectra of miRNAs involved in EMT. A list of mutant p53 regulated miRNAs is presented below:

\section{miR-130b}

The mutant p53 control different networks of genes, it is not surprising that they also affect different spectra of miRNAs involved in EMT. For example, mutant p53 exerts oncogenic functions and promotes EMT by directly binding to the promoter of miR-130b (a negative regulator of Zeb1) and inhibiting its transcription. Attenuation of mutant p53 in endometrial cancer cells increased
miR-130b expression, leading to repression of Zeb1 and blocking the execution of the EMT program ${ }^{90}$. In contrast, Jia et al. have shown that miR-130b by targeting the tumor suppressor protein Pten, enhanced multiple drug resistance, proliferation, and tolerance to apoptosis of breast cancer cells thereby promoting oncogenesis ${ }^{91}$. The nature of this controversy is currently unknown and requires additional investigation.

Interestingly, lncRNA CCAT1, known to interact with p53, also acts as a ceRNA for miR-130b, augmenting the EMT, cell migration, and invasion ${ }^{92}$. By inhibiting the action of miR-130b, CCAT1 upregulates the expression of Zeb1 and Stat 3 in ovarian cancer. While CCAT1 has been observed to be oncogenic in TNBC as well, its association with miR-130b has not been examined in breast cancer yet.

\section{miR-142-3p}

An onco-suppressive miR-142-3p inhibits the proliferation, migration, and invasion of breast cancer cells ${ }^{93}$. It is often downregulated upon overexpression of gain-of-function mutant form of p53 due to hypermethylation of its promoter $^{94}$. Using luciferase reporter assay, it was shown that miR-142-3p interacts with the $3^{\prime} \mathrm{UTR}$ region of Zeb1 mRNA and reduces the levels of Zeb1 transcript and protein. This result suggests that miR-142 likely represses EMT.

Multiple lncRNAs are able to counteract the EMTsuppressing effects of miR-142. In HCC, the RNA product of the taurine upregulated gene 1 (TUG1) reduces the effect of miR-142-3p on Zeb1 by competitively binding to miR-142-3p, acting as ceRNA ${ }^{95}$. Knockdown of IncRNATUG1 was consistent with an increase in miR-142-3p levels and limited the invasion of cells. In breast cancer, it was revealed that another lncRNA NNT-AS1, functions as ceRNA specific for miR-142-3p, thereby restoring Zeb1 and blocking $\mathrm{EMT}^{96}$. IncRNA NNT-AS1 was also significantly overexpressed in breast cancer and correlated with poor prognosis.

\section{miR-218}

Expression of another miR-218 is specifically upregulated by mutant $\mathrm{p} 53(\mathrm{R} 172 \mathrm{H})$ in mesenchymal cells ${ }^{67}$. The $\mathrm{R} 172 \mathrm{H}$ mutant displays a GOF activity during the reprogramming of somatic cells into induced pluripotent stem cells and also increases their oncogenic potential. While miR-218 was upregulated in mutant p53 cells and downregulated in wtp53, the exact role of miR-218 in facilitating this reprogramming has not been detailed yet. Unexpectedly, miR-218 was shown to regulate EMT by inhibiting the expression of Zeb2. Liu et al. investigated the role of miR-214 and miR-218 in breast cancer ${ }^{97}$. The authors found that the aberrant expression of miR-214 and miR-218 were negatively associated with $\mathrm{Ki} 67$, and the expression of miR-218 expression was positively associated with progesterone receptor (PR) in breast 
cancer tissues. Upon overexpression, the cell proliferation and migration in vitro were decreased and cell apoptosis was induced in breast cancer cells. The authors concluded that miR-214 and miR-218 function as tumor suppressors in breast cancer.

miR-218 also caused the inhibition of tumor growth and metastasis in lung cancer. Taken together, these reports may suggest that exerts different functions depending on the cellular context, i.e. in epithelial cells it serves as a tumor suppressor and in the mesenchymal ones upon the induction of pluripotency it may behave as an oncogene.

\section{Conclusions}

MicroRNAs play a decisive role in EMT, either as effector molecules of major transcription factors or as modulators of their expression. Recently, it has become apparent that miRNAs are among the critical regulators of EMT, with the miR-200 family making the main contribution to the process. This in no way undermines the relevance of other players, however, it should be noted that miRNAs play an important role in the regulation of TFs genes, whose products are master-regulators of the EMT. In addition, due to the large number of miRNAs operating in this process, it seems that the regulation is carried out by the additive principle: even a slight dysregulation in the expression levels of several members of one miRNA family would lead to significant amplification of the effect on the level of protein expression for Zeb/Snail/Twist, subsequently affecting the course of EMT. The regulatory complexity of EMT is further exacerbated by the fact that miRNAs themselves are often regulated by other lncRNAs. These feedback loops and networks of p53 and the EMT TFs, miRNA, and lncRNAs involved in the regulation of EMT are shown in Figs. 1 and 2.

Over the past few years, there has been an increase in the number of articles on the regulation of miRNAs using lncRNA. Based on this, it is likely that in the near future, the list of known miRNA regulators involved in EMT will expand significantly. The p53 tumor suppressor protein is a transcription factor by itself. Thus, perhaps it was not surprising to find out that p53 regulates vital cellular processes, including EMT, by regulating multiple miRNAs. Given the fact that p53 is mutated in more than $50 \%$ of all human cancers, the question arises as to whether the mutant p53 regulates different cohorts of miRNAs. In fact, it can be hypothesized that the "onco-miRs" regulated by various mutants of p53 may facilitate EMT, in contrast to wild-type p53. Thus, in order to unravel the complex network of EMT regulation one would take into account the status of p53 and other major tumor suppressors as they may all affect the final outcome of EMT and its reverse process called MET. This knowledge should provide means to consciously intervene with the regulation of EMT as part of the anti-cancer therapy.

\section{Acknowledgements}

N.B., O.F., A.D., and R.K. would like to acknowledge the joint funding from Russian Science Foundation (RSF grant \#19-45-02011), Russia and Department of Science and Technology (DST), India. S.P., O.F., A.D., and N.B. acknowledge Mega-grant program of the Government of Russian Federation (\#14. W03.31.0029). N.B., O.F., and A.D. also appreciate the support of RFBR (grant \#18-29-09144). A.S. thanks Council of Scientific and Industrial Research (CSIR), Govt. of India for Junior Research Fellowship.

\section{Author details \\ ${ }^{1}$ Institute of Cytology RAS, Saint-Petersburg 194064, Russia. ${ }^{2}$ Department of Biochemical Engineering and Biotechnology, Indian Institute of Technology, Delhi 110016, India. ${ }^{3}$ Almazov Federal North-West Medical Research Centre, St- Petersburg, Russia. ${ }^{4}$ Moscow Institute of Physics and Technology, Dolgoprudny 141701, Russia. ${ }^{5}$ Orekhovich Institute of Medical Biochemistry, Moscow 119435, Russia}

\section{Author contributions}

S.P. and A.S. collected the data and wrote the manuscript. N.B. and R.K. designed and edited the manuscript. O.F. designed illustrations. A.D. collected the data for Tables 1-3.

\section{Conflict of interest}

The authors declare that they have no conflict of interest.

\section{Publisher's note}

Springer Nature remains neutral with regard to jurisdictional claims in published maps and institutional affiliations.

Received: 13 September 2020 Revised: 2 December 2020 Accepted: 4 December 2020

Published online: 04 January 2021

\section{References}

1. Redig, A. J. \& McAllister, S. S. Breast cancer as a systemic disease: a view of metastasis. J. Intern. Med. 274, 113-126 (2013).

2. Nassar, F. J., Nasr, R. \& Talhouk, R. MicroRNAs as biomarkers for early breast cancer diagnosis, prognosis and therapy prediction. Pharmacol. Ther. 172, 34-49 (2017).

3. Huang, Q.-Y. et al. Long non-coding RNA: dual effects on breast cancer metastasis and clinical applications. Cancers 11, 1802 (2019).

4. Kim, T. et al. p53 regulates epithelial-mesenchymal transition through microRNAs targeting ZEB1 and ZEB2. J. Exp. Med. 208, 875-883 (2011).

5. Frisch, S. M., Schaller, M. \& Cieply, B. Mechanisms that link the oncogenic epithelial-mesenchymal transition to suppression of anoikis. J. Cell Sci. 126, 21-29 (2013).

6. Kurrey, N. K. et al. Snail and slug mediate radioresistance and chemoresistance by antagonizing p53-mediated apoptosis and acquiring a stem-like phenotype in ovarian cancer cells. Stem Cells 27, 2059-2068 (2009).

7. Tiwari, V. Burn wound: how it differs from other wounds?. Indian J. Plast. Surg. 45, 364-373 (2012).

8. Gugnoni, M. \& Ciarrocchi, A. Long noncoding RNA and epithelial mesenchymal transition in cancer. Int. J. Mol. Sci. 20, 1924 (2019).

9. Chaffer, C. L. et al. Poised chromatin at the ZEB1 promoter enables breast cancer cell plasticity and enhances tumorigenicity. Cell 154, 61-74 (2013).

10. Eger, A. et al. DeltaEF1 is a transcriptional repressor of E-cadherin and regulates epithelial plasticity in breast cancer cells. Oncogene 24, 2375-2385 (2005).

11. Comijn, J. et al. The two-handed $\mathrm{E}$ box binding zinc finger protein SIP1 downregulates E-cadherin and induces invasion. Mol. Cell 7, 1267-1278 (2001).

12. Hajra, K. M., Chen, D. Y. \& Fearon, E. R. The SLUG zinc-finger protein represses E-cadherin in breast cancer. Cancer Res. 62, 1613-1618 (2002).

13. Yang, J. et al. Twist, a master regulator of morphogenesis, plays an essential role in tumor metastasis. Cell 117, 927-939 (2004).

14. Muenst, $\mathrm{S}$. et al. Nuclear expression of snail is an independent negative prognostic factor in human breast cancer. Dis. Markers 35, 337-344 (2013). 
15. Shi, J. et al. Disrupting the interaction of BRD4 with diacetylated twist suppresses tumorigenesis in basal-like breast cancer. Cancer Cell 25, 210-225 (2014).

16. Li, Y. et al. Slug contributes to cancer progression by direct regulation of ERa signaling pathway. Int. J. Oncol. 46, 1461-1472 (2015).

17. Storci, G. et al. The basal-like breast carcinoma phenotype is regulated by SLUG gene expression. J. Pathol. 214, 25-37 (2008).

18. Lane, D. P. Cancer. p53, guardian of the genome. Nature 358, 15-16 (1992)

19. Bieging, K. T., Mello, S. S. \& Attardi, L. D. Unravelling mechanisms of p53mediated tumour suppression. Nat. Rev. Cancer 14, 359-370 (2014).

20. Rinon, A. et al. p53 coordinates cranial neural crest cell growth and epithelialmesenchymal transition/delamination processes. Development $\mathbf{1 3 8}$ 1827-1838 (2011).

21. Yang-Hartwich, Y. et al. p53-Pirh2 complex promotes Twist1 degradation and inhibits EMT. Mol. Cancer Res. 17, 153-164 (2019).

22. $\mathrm{Ni}$, T. et al. Snail1-dependent $\mathrm{p} 53$ repression regulates expansion and activity of tumour-initiating cells in breast cancer. Nat. Cell Biol. 18, 1221-1232 (2016).

23. Chang, C.-J. et al. p53 regulates epithelial-mesenchymal transition and stem cell properties through modulating miRNAs. Nat. Cell Biol. 13, 317-323 (2011).

24. Dong, P. et al. Mutant p53 gain-of-function induces epithelial-mesenchymal transition through modulation of the miR-130b-ZEB1 axis. Oncogene $\mathbf{3 2}$ 3286-3295 (2013).

25. Fedorova, $\mathrm{O}$. et al. Attenuation of p53 mutant as an approach for treatment Her2-positive cancer. Cell Death Discov. 6, 1-8 (2020).

26. Puisieux, A., Brabletz, T. \& Caramel, J. Oncogenic roles of EMT-inducing transcription factors. Nat. Cell Biol. 16, 488-494 (2014).

27. Gregory, P. A. et al. The miR-200 family and miR-205 regulate epithelial to mesenchymal transition by targeting ZEB1 and SIP1. Nat. Cell Biol. 10 593-601 (2008).

28. Yan, X. et al. Mesenchymal stem cells promote hepatocarcinogenesis via IncRNA-MUF interaction with ANXA2 and miR-34a. Cancer Res. 77 6704-6716 (2017).

29. Burk, U. et al. A reciprocal repression between ZEB1 and members of the miR-200 family promotes EMT and invasion in cancer cells. EMBO Rep. 9, 582-589 (2008).

30. Li, C. et al. Long non-coding RNA XIST promotes TGF-B-induced epithelialmesenchymal transition by regulating miR-367/141-ZEB2 axis in non-smallcell lung cancer. Cancer Lett. 418, 185-195 (2018).

31. Shen, J. et al. LnCRNA XIST promotes pancreatic cancer migration, invasion and EMT by sponging miR-429 to modulate ZEB1 expression. Int. J. Biochem. Cell Biol. 113, 17-26 (2019).

32. Su, X. et al. microRNAs and ceRNAs: RNA networks in pathogenesis of cancer. Chin. J. Cancer Res. 25, 235 (2013)

33. Zhou, W. et al. The IncRNA H19 mediates breast cancer cell plasticity during EMT and MET plasticity by differentially sponging miR-200b/c and let-7b. Sci. Signal. 10, eaak9557 (2017).

34. Zhang, L., Liao, Y. \& Tang, L. MicroRNA-34 family: a potential tumor suppressor and therapeutic candidate in cancer. J. Exp. Clin. Cancer Res. 38, 53 (2019).

35. Raver-Shapira, N. et al. Transcriptional activation of miR-34a contributes to p53-mediated apoptosis. Mol. Cell 26, 731-743 (2007)

36. Siemens, $\mathrm{H}$. et al. miR-34 and SNAlL form a double-negative feedback loop to regulate epithelial-mesenchymal transitions. Cell Cycle 10,4256-4271 (2011).

37. Imani, S. et al. MicroRNA-34a targets epithelial to mesenchymal transitioninducing transcription factors (EMT-TFs) and inhibits breast cancer cell migration and invasion. Oncotarget 8, 21362 (2017).

38. De Carolis, $\mathrm{S}$. et al. Carbonic anhydrase $9 \mathrm{mRNA} / \mathrm{microRNA34a}$ interplay in hypoxic human mammospheres. J. Cell. Physiol. 231, 1534-1541 (2016).

39. Song, P., Ye, L. F., Zhang, C., Peng, T. \& Zhou, X. H. Long non-coding RNA XIST exerts oncogenic functions in human nasopharyngeal carcinoma by targeting miR-34a-5p. Gene 592, 8-14 (2016).

40. Ji, Y., Wang, M., Li, X. \& Cui, F. The long noncoding RNA NEAT1 targets miR$34 a-5 p$ and drives nasopharyngeal carcinoma progression via $W n t / \beta$-catenin signaling. Yonsei Med. J. 60, 336-345 (2019).

41. Kim, T. et al. p53 regulates epithelial-mesenchymal transition through microRNAs targeting ZEB1 and ZEB2. J. Exp. Med. 208, 875-883 (2011).

42. Gao, J. B., Zhu, M. N. \& Zhu, X. L. miRNA-215-5p suppresses the aggressiveness of breast cancer cells by targeting Sox9. FEBS Open Bio 9, 1957-1967 (2019).
43. Kong, $X$. et al. LncRNA-CDC6 promotes breast cancer progression and function as ceRNA to target CDC6 by sponging microRNA-215. J. Cell. Physiol. 234, 9105-9117 (2019)

44. di Gennaro, A. et al. A p53/miR-30a/ZEB2 axis controls triple negative breast cancer aggressiveness. Cell Death Differ. 25, 2165-2180 (2018).

45. Liu, Y. et al. Linc HOTAIR epigenetically silences miR34a by binding to PRC2 to promote the epithelial-to-mesenchymal transition in human gastric cancer. Cell Death Dis. 6, e1802-e1802 (2015).

46. Rahimi, M. et al. Down-regulation of miR-200c and up-regulation of miR-30c target both stemness and metastasis genes in breast cancer. Cell J. $\mathbf{2 1}$ 467-478 (2020)

47. Feng, $\mathrm{T}$. et al. MiR-124 inhibits cell proliferation in breast cancer through downregulation of CDK4. Tumour Biol. 36, 5987-5997 (2015).

48. Liang, Y. J. et al. MiR-124 targets Slug to regulate epithelial-mesenchymal transition and metastasis of breast cancer. Carcinogenesis 34, 713-722 (2013).

49. Zhou, $H$. et al. LncRNA HOTAIR promotes renal interstitial fibrosis by regulating Notch1 pathway via the modulation of miR-124. Nephrology 24, 472-480 (2019).

50. Zhang, Z. et al. Epigenetic silencing of miR-203 upregulates SNAI2 and contributes to the invasiveness of malignant breast cancer cells. Genes Cancer 2. 782-791 (2011)

51. Ding, X., Park, S. I., McCauley, L. K. \& Wang, C. Y. Signaling between transforming growth factor $\beta$ (TGF- $\beta$ ) and transcription factor SNAI2 represses expression of microRNA miR-203 to promote epithelial-mesenchymal transition and tumor metastasis. J. Biol. Chem. 288, 10241-10253 (2013).

52. Chen, J., Zhong, Y. \& Li, L. miR-124 and miR-203 synergistically inactivate EMT pathway via coregulation of ZEB2 in clear cell renal cell carcinoma (cCRCC). J. Transl. Med. 18, 69 (2020).

53. McKenna, D. J., McDade, S. S., Patel, D. \& McCance, D. J. MicroRNA 203 expression in keratinocytes is dependent on regulation of p53 levels by E6. J. Virol. 84, 10644-10652 (2010).

54. Funamizu, N., Lacy, C. R., Kamada, M., Yanaga, K. \& Manome, Y. MicroRNA-203 induces apoptosis by upregulating Puma expression in colon and lung cancer cells. Int. J. Oncol. 47, 1981-1988 (2015)

55. Wang, L. et al. Long noncoding RNA B3GALT5-AS1 suppresses colon cancer liver metastasis via repressing microRNA-203. Aging 10, 3662-3682 (2018).

56. Tan, G. et al. A novel role for microRNA-129-5p in inhibiting ovarian cancer cell proliferation and survival via direct suppression of transcriptional Coactivators YAP and TAZ. Oncotarget 6, 8676-8686 (2015).

57. Xiao, L. et al. MicroRNA-129-5p modulates epithelial-to-mesenchymal transition by targeting SIP1 and SOX4 during peritoneal dialysis. Lab. Invest. 95 817-832 (2015)

58. $Y u, Y$. et al. Down-regulation of miR-129-5p via the Twist1-Snail feedback loop stimulates the epithelial-mesenchymal transition and is associated with poor prognosis in breast cancer. Oncotarget 6, 34423-34436 (2015).

59. Yao, N. et al. Long non-coding RNA NONHSAT101069 promotes epirubicin resistance, migration, and invasion of breast cancer cells through NONHSAT101069/miR-129-5p/Twist1 axis. Oncogene 38, 7216-7233 (2019).

60. Zuo, Y., Li, Y., Zhou, Z., Ma, M. \& Fu, K. Long non-coding RNA MALAT1 promotes proliferation and invasion via targeting miR-129-5p in triplenegative breast cancer. Biomed. Pharmacother. 95, 922-928 (2017).

61. Wu, Y. et al. The role of IncRNAs in the distant metastasis of breast cancer. Front. Oncol. 9, 407 (2019).

62. Pruszko, M. et al. The mutant p53-ID4 complex controls VEGFA isoforms by recruiting IncRNA MALAT1. EMBO Rep. 18, 1331-1351 (2017).

63. Herrera-Merchan, A. et al. miR-33-mediated downregulation of p53 controls hematopoietic stem cell self-renewal. Cell Cycle 9, 3277-3285 (2010).

64. $\mathrm{Wu}, \mathrm{Q}$. et al. Long non-coding RNA CASC15 regulates gastric cancer cell proliferation, migration and epithelial mesenchymal transition by targeting CDKN1A and ZEB1. Mol. Oncol. 12, 799-813 (2018).

65. Zhang, $C$. et al. MiR-33a suppresses breast cancer cell proliferation and metastasis by targeting ADAM9 and ROS1. Protein Cell 6, 881-889 (2015).

66. Lin, Y. et al. MicroRNA-33b inhibits breast cancer metastasis by targeting HMGA2, SALL4 and Twist1. Sci. Rep. 5, 9995 (2015).

67. Grespi, F. et al. Differential regulated microRNA by wild type and mutant p53 in induced pluripotent stem cells. Cell Death Dis. 7, e2567 (2016).

68. Guo, F. et al. MiR-101 suppresses the epithelial-to-mesenchymal transition by targeting ZEB1 and ZEB2 in ovarian carcinoma. Oncol. Rep. 31, 2021-2028 (2014). 
69. Meng, Q., Yang, B. Y., Liu, B., Yang, J. X. \& Sun, Y. Long non-coding RNA SNHG6 promotes glioma tumorigenesis by sponging miR-101-3p. Int. J. Biol. Markers 33, 148-155 (2018)

70. Cheng, Y. et al. LncRNA XIST promotes the epithelial to mesenchymal transition of retinoblastoma via sponging miR-101. Eur. J. Pharmacol. 843, 210-216 (2019).

71. Liang, $H$. et al. LncRNA PTAR promotes EMT and invasion-metastasis in serous ovarian cancer by competitively binding miR-101-3p to regulate ZEB1 expression. Mol. Cancer 17, 119 (2018).

72. Piovan, $C$. et al. Oncosuppressive role of p53-induced miR-205 in triple negative breast cancer. Mol. Oncol. 6, 458-472 (2012).

73. Lee, J. Y. et al. Loss of the polycomb protein Mel-18 enhances the epithelialmesenchymal transition by ZEB1 and ZEB2 expression through the downregulation of miR-205 in breast cancer. Oncogene 33, 1325-1335 (2014).

74. Wang, X. et al. Upregulation of MiR-205 under hypoxia promotes epithelialmesenchymal transition by targeting ASPP2. Cell Death Dis. 7, e2517 (2016).

75. Hou, P. et al. LincRNA-ROR induces epithelial-to-mesenchymal transition and contributes to breast cancer tumorigenesis and metastasis. Cell Death Dis. $\mathbf{5}$ e1287 (2014).

76. Song, S. et al. LncRNA ADPGK-AS1 promotes pancreatic cancer progression through activating ZEB1-mediated epithelial-mesenchymal transition. Cancer Biol. Ther. 19, 573-583 (2018).

77. Piva, R., Spandidos, D. A. \& Gambari, R. From microRNA functions to microRNA therapeutics: novel targets and novel drugs in breast cancer research and treatment (review). Int. J. Oncol. 43, 985-994 (2013).

78. Lambertini, E. et al. Correlation between Slug transcription factor and miR221 in MDA-MB-231 breast cancer cells. BMC Cancer 12, 445 (2012).

79. Fornari, F. et al. p53/mdm2 feedback loop sustains miR-221 expression and dictates the response to anticancer treatments in hepatocellular carcinoma. Mol. Cancer Res. 12, 203-216 (2014)

80. Jin, $X$. et al. CASC2/miR-24/miR-221 modulates the TRAlL resistance of hepatocellular carcinoma cell through caspase-8/caspase-3. Cell Death Dis. 9 318 (2018).

81. Arora, H., Qureshi, R. \& Park, W. Y. miR-506 regulates epithelial mesenchyma transition in breast cancer cell lines. PLoS One 8, e64273 (2013).

82. Yin, M. et al. Selective killing of lung cancer cells by miRNA-506 molecule through inhibiting NF-KB p65 to evoke reactive oxygen species generation and p53 activation. Oncogene 34, 691-703 (2015)

83. Wang, X. X. et al. miR-506 attenuates methylation of IncRNA MEG3 to inhibit migration and invasion of breast cancer cell lines via targeting SP1 and SP3. Cancer Cell Int. 18, 171 (2018).

84. Sun, L., Li, Y. \& Yang, B. Downregulated long non-coding RNA MEG3 in breast cancer regulates proliferation, migration and invasion by depending on p53's transcriptional activity. Biochem. Biophys. Res. Commun. 478 323-329 (2016).

85. Yong, W. et al. Long noncoding RNA NEAT1, regulated by LIN28B, promotes cell proliferation and migration through sponging miR-506 in high-grade serous ovarian cancer. Cell Death Dis. 9, 861 (2018).

86. Dugimont, T. et al. The H19 TATA-less promoter is efficiently repressed by wild-type tumor suppressor gene product p53. Oncogene 16, 2395-2401 (1998).

87. Matouk, I. J. et al. Oncofetal H19 RNA promotes tumor metastasis. Biochim. Biophys. Acta 1843, 1414-1426 (2014).

88. Ma, L., Teruya-Feldstein, J. \& Weinberg, R. A. Tumour invasion and metastasis initiated by microRNA-10b in breast cancer. Nature 449, 682-688 (2007).

89. Bisio, A. et al. Identification of new p53 target microRNAs by bioinformatics and functional analysis. BMC Cancer 13, 552 (2013).

90. Dong, P. et al. Mutant p53 gain-of-function induces epithelial-mesenchymal transition through modulation of the miR-130b-ZEB1 axis. Oncogene $\mathbf{3 2}$ 3286-3295 (2013).

91. Miao, Y. et al. MicroRNA-130b targets PTEN to mediate drug resistance and proliferation of breast cancer cells via the PI3K/Akt signaling pathway. Sci. Rep. 7, 41942 (2017).

92. Cao, Y., Shi, H., Ren, F., Jia, Y. \& Zhang, R. Long non-coding RNA CCAT1 promotes metastasis and poor prognosis in epithelial ovarian cancer. Exp. Cell Res. 359, 185-194 (2017).

93. Schwickert, A. et al. microRNA miR-142-3p inhibits breast cancer cell invasiveness by synchronous targeting of WASL, Integrin Alpha V, and additional cytoskeletal elements. PLoS ONE 10, e0143993 (2015)

94. Godfrey, J. D., Morton, J. P., Wilczynska, A., Sansom, O. J. \& Bushell, M. D. MiR$142-3 p$ is downregulated in aggressive p53 mutant mouse models of pancreatic ductal adenocarcinoma by hypermethylation of its locus. Cell Death Dis. 9, 644 (2018).

95. He, C. et al. IncRNA TUG1-mediated Mir-142-3p downregulation contributes to metastasis and the epithelial-to-mesenchymal transition of hepatocellular carcinoma by targeting ZEB1. Cell. Physiol. Biochem. 48, 1928-1941 (2018).

96. Li, Y. et al. Long non-coding RNA NNT-AS1 affects progression of breast cancer through miR-142-3p/ZEB1 axis. Biomed. Pharmacother. 103, 939-946 (2018).

97. Liu, B. et al. Tumor-suppressing roles of miR-214 and miR-218 in breast cancer. Oncol. Rep. 35, 3178-3184 (2016).

98. $\mathrm{Xu}$, T. et al. MiR-142-3p functions as a tumor suppressor by targeting RAC1/ PAK1 pathway in breast cancer. J. Cell. Physiol. 235, 4928-4940 (2020).

99. Troschel, F. M. et al. miR-142-3p attenuates breast cancer stem cell characteristics and decreases radioresistance in vitro. Tumor Biol. 40 1010428318791887 (2018).

100. Song, C. et al. High expression of microRNA-183/182/96 cluster as a prognostic biomarker for breast cancer. Sci. Rep. 6, 24502 (2016).

101. Lowery, A. J., Miller, N., Dwyer, R. M. \& Kerin, M. J. Dysregulated miR-183 inhibits migration in breast cancer cells. BMC Cancer 10, 502 (2010).

102. Hong, Y. et al. miR-96 promotes cell proliferation, migration and invasion by targeting PTPN9 in breast cancer. Sci. Rep. 6, 37421 (2016).

103. Antolín, S. et al. Circulating miR-200c and miR-141 and outcomes in patients with breast cancer. BMC Cancer 15, 297 (2015)

104. Choi, S. K. et al. Overexpression of the miR-141/200c cluster promotes the migratory and invasive ability of triple-negative breast cancer cells through the activation of the FAK and PI3K/AKT signaling pathways by secreting VEGF-A. BMC Cancer 16, 570 (2016).

105. Debeb, B. G. et al. miR-141-mediated regulation of brain metastasis from breast cancer. J. Natl. Cancer Inst. 108, djw026 (2016).

106. Madhavan, D. et al. Circulating miRNAs as surrogate markers for circulating tumor cells and prognostic markers in metastatic breast cancer. Clin. Cancer Res. 18, 5972-5982 (2012).

107. D'Ippolito, E. et al. miR-9 and miR-200 regulate PDGFRß-mediated endothelial differentiation of tumor cells in triple-negative breast cancer. Cancer Res. 76 5562-5572 (2016).

108. Castilla, M. A. et al. MicroRNA-200 family modulation in distinct breast cancer phenotypes. PLOS ONE 7, e47709 (2012).

109. Yang, F., Zhang, W., Shen, Y. \& Guan, X. Identification of dysregulated microRNAs in triple-negative breast cancer. Int. J. Oncol. 46, 927-932 (2015)

110. Tuomarila, M. et al. Overexpression of microRNA-200c predicts poor outcome in patients with PR-negative breast cancer. PLOS ONE 9, e109508 (2014).

111. Li, D. et al. The microRNAs miR-200b-3p and miR-429-5p target the LIMK1/ CFL1 pathway to inhibit growth and motility of breast cancer cells. Oncotarget 8, 85276 (2017).

112. Wang, C., Ju, H., Shen, C. \& Tong, Z. miR-429 mediates $\delta$-tocotrienol-induced apoptosis in triple-negative breast cancer cells by targeting XIAP. Int. J. Clin. Exp. Med. 8, 15648 (2015).

113. Ye, Z.-B. et al. miR-429 inhibits migration and invasion of breast cancer cells in vitro. Int. J. Oncol. 46, 531-538 (2015).

114. Weihua, Z., Guorong, Z., Xiaolong, C. \& Weizhan, L. MiR-33a functions as a tumor suppressor in triple-negative breast cancer by targeting EZH2. Cancer Cell Int. 20, 1-12 (2020)

115. LV, Z. D. et al. miR-655 suppresses epithelial-to-mesenchymal transition by targeting Prrx1 in triple-negative breast cancer. J. Cell. Mol. Med. 20, 864-873 (2016).

116. Li, W., Zhai, L., Zhao, C. \& LV, S. miR-153 inhibits epithelial-mesenchymal transition by targeting metadherin in human breast cancer. Breast Cancer Res. Treat. 150, 501-509 (2015).

117. Liu, R. et al. Mifepristone suppresses basal triple-negative breast cancer stem cells by down-regulating KLF5 expression. Theranostics 6, 533 (2016).

118. Tavakolian, S., Goudarzi, H., Torfi, F. \& Faghihloo, E. Evaluation of microRNA-9 and-192 expression levels as biomarkers in patients suffering from breast cancer. Biomed. Rep. 12, 30-34 (2020).

119. Chen, P. et al. MicroRNA-192 inhibits cell proliferation and induces apoptosis in human breast cancer by targeting caveolin 1. Oncol. Rep. 42, 1667-1676 (2019).

120. Gao, J. B., Zhu, M. N. \& Zhu, X. L. miRNA-215-5p suppresses the aggressiveness of breast cancer cells by targeting Sox9. FEBS Open Bio 9, 1957-1967 (2019)

121. Zhou, S.-w et al. Aberrant miR-215 expression is associated with clinical outcome in breast cancer patients. Med. Oncol. 31, 259 (2014). 
122. Ahmadinejad, F. et al. Lower expression of miR-218 in human breast cancer is associated with lymph node metastases, higher grades, and poorer prognosis. Tumor Biol. 39, 1010428317698362 (2017).

123. Jin, Y. et al. MicroRNA-338-3p functions as tumor suppressor in breast cancer by targeting SOX4. Int. J. Oncol. 47, 1594-1602 (2015).

124. Wang, R. et al. MiR-101 is involved in human breast carcinogenesis by targeting Stathmin1. PLOS ONE 7, e46173 (2012).

125. Wu, H., Zhu, S. \& Mo, Y.-Y. Suppression of cell growth and invasion by miR205 in breast cancer. Cell Res. 19, 439-448 (2009).

126. Xiao, Y. et al. Integrin a5 down-regulation by miR-205 suppresses triple negative breast cancer stemness and metastasis by inhibiting the SrcNav2/ Rac1 pathway. Cancer Lett. 433, 199-209 (2018).

127. Raychaudhuri, M. et al. MicroRNAs miR-7 and miR-340 predict response to neoadjuvant chemotherapy in breast cancer. Breast Cancer Res. Treat. 162, 511-521 (2017).

128. Shi, Z. et al. MiR-340 inhibits triple-negative breast cancer progression by reversing EZH2 mediated miRNAs dysregulated expressions. J. Cancer 8, 3037 (2017).

129. Li, Q. et al. Involvement of NF-K B/miR-448 regulatory feedback loop in chemotherapy-induced epithelial-mesenchymal transition of breast cancer cells. Cell Death Differ. 18, 16-25 (2011).

130. Guo, S. et al. MiR-508-3p inhibits cell invasion and epithelial-mesenchymal transition by targeting ZEB1 in triple-negative breast cancer. Eur. Rev. Med. Pharm. Sci. 22, 6379-6385 (2018).

131. Cheng, C.-W. et al. MicroRNA-30a inhibits cell migration and invasion by downregulating vimentin expression and is a potential prognostic marker in breast cancer. Breast Cancer Res. Treat. 134, 1081-1093 (2012).

132. Kawaguchi, T. et al. Overexpression of suppressive microRNAs, miR-30a and miR-200c are associated with improved survival of breast cancer patients. Sci. Rep. 7, 1-12 (2017).

133. Tanic, M. et al. Deregulated miRNAs in hereditary breast cancer revealed a role for miR-30c in regulating KRAS oncogene. PLOS ONE 7 e38847 (2012).

134. Dobson, J. R. et al. hsa-mir-30c promotes the invasive phenotype of metastatic breast cancer cells by targeting NOV/CCN3. Cancer Cell Int. 14, 73 (2014)
135. Peurala, H. et al. MiR-34a expression has an effect for lower risk of metastasis and associates with expression patterns predicting clinical outcome in breast cancer. PLOS ONE 6, e26122 (2011).

136. Zeng, Z., Chen, X., Zhu, D., Luo, Z. \& Yang, M. Low expression of circulating microRNA-34C is associated with poor prognosis in triple-negative breast cancer. Yonsei Med. J. 58, 697-702 (2017).

137. Sandhu, R., Rivenbark, A. G., Mackler, R. M., Livasy, C. A. \& Coleman, W. B. Dysregulation of microRNA expression drives aberrant DNA hypermethylation in basal-like breast cancer. Int. J. Oncol. 44, 563-572 (2014).

138. Li, L. et al. Microrna-124 targets flotillin-1 to regulate proliferation and migration in breast cancer. Mol. Cancer 12, 163 (2013).

139. Zhang, T.-F. M., Ji, H., LV, M.-M., Zhao, J.-H. \& Tang, J.-H. Down-regulation of miRNA-452 is associated with adriamycin-resistance in breast cancer cells. Asian Pac. J. Cancer Prev. 15, 5137-5142 (2014).

140. Kim, M. et al. VEGFA links self-renewal and metastasis by inducing Sox2 to repress miR-452, driving Slug. Oncogene 36, 5199-5211 (2017).

141. Wang, S., Li, H., Wang, J. \& Wang, D. Retracted article: expression of microRNA-497 and its prognostic significance in human breast cancer. Diagn. Pathol. 8, 172 (2013).

142. Sun, G., Liu, Y., Wang, K. \& Xu, Z. miR-506 regulates breast cancer cell metastasis by targeting IQGAP1. Int. J. Oncol. 47, 1963-1970 (2015).

143. Liu, $\mathrm{H}$. et al. Expression and regulatory function of miRNA-182 in triplenegative breast cancer cells through its targeting of profilin 1. Tumor Biol. 34 1713-1722 (2013).

144. Wang, P. Y. et al. Higher expression of circulating miR-182 as a novel biomarker for breast cancer. Oncol. Lett. 6, 1681-1686 (2013).

145. Lu, X. et al. MiR-129-5p sensitizes the response of Her-2 positive breast cancer to trastuzumab by reducing Rps6. Cell. Physiol. Biochem. 44, 2346-2356 (2017).

146. Meng, R. et al. miR-129-5p suppresses breast cancer proliferation by targeting CBX4. Neoplasma 65, 572-578 (2018).

147. Luo, J., Chen, J. \& He, L. mir-129-5p attenuates irradiation-induced autophagy and decreases radioresistance of breast cancer cells by targeting HMGB1 Med. Sci. Monit. 21, 4122 (2015).

148. Majumder, M., Landman, E., Liu, L., Hess, D. \& Lala, P. K. COX-2 elevates oncogenic miR-526b in breast cancer by EP4 activation. Mol. Cancer Res. 13 1022-1033 (2015). 